\title{
Aggregation of nanoscale iron oxyhydroxides and corresponding effects on Zn(II) uptake, retention, and speciation
}

\begin{abstract}
C.S. KIM ${ }^{1}$
${ }^{1}$ Chapman University, Orange, CA USA; cskim@chapman.edu

Nanoscale iron oxyhydroxides, which are commonly found in surface aqueous systems, act as effective sorbents for dissolved metals due to their chemical reactivity, small size and high surface area which allow them to sequester metal ions from the aqueous and into the solid phase. However, they also rapidly aggregate under natural geochemical conditions, thus affecting their metal sorption and retention capabilities. The broad range of particle aggregation mechanisms and states possible in the environment, in addition to variations in exposure/reaction time and other aqueous conditions, substantially increases the complexity and variety of interactions between metal ions and nanoparticle aggregates.

We have conducted an array of macroscopic batch uptake experiments and spectroscopic analyses using synchrotron Xrays to explore $\mathrm{Zn}$ (II) adsorption, desorption, and retention processes associated with both dispersed and aggregated iron oxyhydroxide nanoparticles, with a range of aggregation states induced by partial/complete suspension freezing, suspension drying, and the presence of humic acid (1-250 $\mathrm{mg} / \mathrm{L}$ ). Nanoparticle aggregate state was assessed by dynamic light scattering (DLS), Zn(II) adsorption/retention behavior through macroscopic batch experiments and inductively coupled plasma-optical emission spectrometry (ICP-OES), and $\mathrm{Zn}$ (II) speciation using extended X-ray absorption fine structure (EXAFS) spectroscopy.

Nanoparticle aggregation state was found to result in reduced initial $\mathrm{Zn}$ (II) uptake, likely due to lowered surface area, but in increased metal retention, likely due to interparticle nanoporosity. Quantifiable variations in zinc speciation at the solution-solid interface indicate that a range of $\mathrm{Zn}$ (II) sorption complexes exists at the nanoparticle-water interface, with the more weakly-bound complexes removed by a pH-based desorption step that leaves behind more strongly-bound species, as primarily evidenced by an increase in iron coordination around the average zinc atom. These dynamic changes in surface complexation inform our understanding of fundamental sorption/desorption processes to nanoparticles and their aggregates as well as the development of potential applied remediation strategies in contaminated systems.
\end{abstract}

